\title{
The Effect of Ivabradine on the Heart Rate and Sympathovagal Balance in Postural Tachycardia Syndrome Patients
}

\author{
Merav Barzilai, M.D. ${ }^{1,2}$, and Giris Jacob, M.D., D.Sc. ${ }^{1,2,3 *}$ \\ 'J. Recanati Autonomic Dysfunction Center and ${ }^{2}$ Medicine F, Tel Aviv "Sourasky" Medical Center, Tel \\ Aviv, Israel and ${ }^{s}$ Faculty of Medicine, Tel Aviv University, Tel Aviv, Israel
}

\begin{abstract}
Background: Postural tachycardia syndrome (POTS) is a common form of chronic orthostatic intolerance. The remarkable increase in heart rate (HR) upon standing is the hallmark of this syndrome. Treatment of POTS patients is challenging and includes drugs that slow the HR. Ivabradine is a selective $\mathrm{I}_{f}$ channel blocker designed to slow the HR, as an anti-anginal agent. In view of its ability to slow the HR, we posited that ivabradine may be an ideal medication for treating POTS patients. This report provides the results of an investigation in which we studied ivabradine's effect on the hemodynamics and sympathovagal balance in POTS patients.

Methods: An open-label trial, without a placebo control, was performed in eight patients with POTS of two years' standing. Characterization of symptoms, hemodynamics, autonomic function tests, and HR and blood pressure (BP) variability were determined while patients were in a supine position and during a 20-minute head-up tilt before and after a single oral dose of $7.5 \mathrm{mg}$ ivabradine.
\end{abstract}

\footnotetext{
Abbreviations: ANS, autonomic nervous system; BP, blood pressure; BPV, blood pressure variability; ECG, electrocardiography; HR, heart rate; HRV, heart rate variability; POTS, postural tachycardia syndrome; RMSSD, root mean square of the successive differences; SA, sinoatrial.

Citation: Barzilai M, Jacob G. The Effect of Ivabradine on the Heart Rate and Sympathovagal Balance in Postural Tachycardia Syndrome Patients. Rambam Maimonides Med J 2015;6 (3):eoo28. doi:10.5041/RMMJ.10213
}

Copyright: (C) 2015 Barzilai and Jacob. This is an open-access article. All its content, except where otherwise noted, is distributed under the terms of the Creative Commons Attribution License (http://creativecommons.org/licenses/by/3.0), which permits unrestricted use, distribution, and reproduction in any medium, provided the original work is properly cited.

Conflict of interest: No potential conflict of interest relevant to this article was reported.

* To whom correspondence should be addressed. E-mail: jacobgi@tlvmc.gov.il 
Results: Ivabradine slowed the HR of POTS patients at rest by $4 \pm 1 \mathrm{bpm}(P<0.05)$. During a 5 -minute head-up tilt, the HR decreased from $118 \pm 4 \mathrm{bpm}$ to $101 \pm 5 \mathrm{bpm}(P<0.01)$. Ivabradine did not affect the BP when patients were at rest in a supine position or in head-up tilt position. Cardiovascular vagal and sympathetic tone, extrapolated from the time and frequency domains of the HR and BP variability, were also not affected by ivabradine.

Conclusions: Ivabradine is an effective drug for slowing the HR of POTS patients at rest and during tilting, without producing significant adverse effects. Moreover, ivabradine exerts its effects without influencing the sympathovagal balance.

KEY WORDS: $I_{\mathrm{f}}$ channel blocker, postural tachycardia syndrome, orthostatic intolerance, sympathovagal balance

\section{INTRODUCTION}

Postural tachycardia syndrome (POTS) is a common form of chronic orthostatic intolerance that occurs on standing and is eventually relieved by lying down or sitting. The syndrome is characterized by a constellation of orthostatic symptoms (which include lightheadedness, dimming of vision, confusion, fatigue, exercise intolerance, and anxiety) and clinical signs (which include a bluish-red skin and edema in dependent limbs). The main physical finding in POTS is the dramatic remarkable increase in heart rate (HR) upon standing ( $>30$ beats per minute (bpm)) without an appreciable decrease in blood pressure (BP). Most POTS patients are women, and the age of presentation is usually between the ages of 15 and 50 years. The quality of life of POTS patients can be substantially reduced to a debilitating level. ${ }^{1-4}$

This syndrome is thought to be a primary adrenergic dysfunction. Most POTS patients have elevated plasma norepinephrine concentrations, hypovolemia, and excessive pooling of blood in the legs while standing. ${ }^{5,6}$ Some studies have shown that sympathetic denervation, especially of the veins in the legs, may be the underlying mechanism of the disease: such denervation results in blood pooling upon standing with reductions in the end-diastolic ventricular volume, and reflex tachycardia. Some POTS patients can present with idiopathic hypovolemia with or without a renin and aldosterone disorder. Other possible causes of POTS are a central hyperadrenergic state, which has a genetic predisposition, and indolent mastocytosis. ${ }^{7-14}$

Secondary POTS can occur in conditions that affect the peripheral autonomic nervous system such as diabetes mellitus, amyloidosis, sarcoidosis, and paraneoplastic syndromes, or following chemotherapy. ${ }^{2,3,9}$
Management of POTS is multifaceted and includes discontinuation of any medications that could contribute to orthostatic intolerance, such as a-adrenoceptor antagonists, diuretics, cGMPspecific phosphodiesterase type 5 inhibitors (sildenafil), and organic nitrates and nitrites, along with identifying those conditions that may cause secondary POTS.

Treating POTS patients comprises nonpharmacological and pharmacological interventions. Conservative measures for patients with mild POTS symptoms include plasma volume expansion and increasing dietary salt and fluid intake. Pharmacotherapy is needed for patients with moderate to severe POTS, and, currently, no drug has been officially approved by the US Food and Drug Administration (FDA) for treating POTS. In fact, most drugs that are given to POTS patients are usually prescribed "off label" and include fludrocortisone, desmopressin, and, sometimes, erythropoietin. Other drugs which can also be therapeutically beneficial in patients with POTS are (1) drugs that increase peripheral vascular resistance such as midodrine, a prodrug whose active metabolite is an a-adrenoceptor antagonist, and somatostatin analogs; (2) drugs that can modify the central and peripheral activity of the sympathetic nervous system such as $\beta$-adrenoceptor antagonists (mainly propranolol), serotonin and norepinephrine uptake inhibitors, and combined $\alpha$ - and $\beta$-adrenoceptor antagonists; and (3) drugs that reduce the sympathetic tone such as clonidine. ${ }^{2,3,9}$

Treatment of POTS is challenging due to the potential for adverse effects from the abovementioned drugs and the limited effectiveness of nonpharmacologic treatments. Many POTS patients have difficulty achieving adequate control of their symptoms. In addition, none of the abovementioned medications are tailored specifically to blunt the 
increase in HR that underlies the many symptoms of POTS.

There is some anecdotal evidence that some POTS patients can be successfully treated with ivabradine, an anti-anginal agent designed to slow the HR. ${ }^{15-26}$ Ivabradine is a selective antagonist of the $\mathrm{I}_{f}$ channel, an ionic current that determines the slope of diastolic depolarization (phase IV action potential). Accordingly, ivabradine controls the time interval between successive action potentials and the HR. Ivabradine also reduces the firing rate of pacemaker cells in the sinoatrial (SA) node, where it mainly influences the intrinsic $\mathrm{HR}$ at concentrations that do not affect other cardiac currents, and has no negative inotropic or lusitropic effects. ${ }^{17,27-32}$ In view of its ability to slow the HR without affecting other cardiovascular functions, we posited that ivabradine may be an ideal medication for treating POTS patients. We report herein on the results of an investigation in which the effect of ivabradine on the hemodynamics and sympathovagal balance of POTS patients was studied.

\section{METHODS}

The investigation was an open-label trial without placebo control. It was approved by the Tel Aviv Sourasky Medical Center Institutional Review Board and was performed according to the principles of the Declaration of Helsinki. The study was done in the J. Recanati Autonomic Dysfunction Center, Department of Internal Medicine F, Tel Aviv "Sourasky" Medical Center, Tel Aviv, Israel. After being provided with an explanation of the purpose, nature, and potential risks of the investigation, each recruited patient signed a consent form.

\section{Subjects}

Inclusion criteria for the investigation were POTS patients with orthostatic intolerance for at least six months; increased HR of at least $30 \mathrm{bpm}$ without a concomitant decrease in BP of more than 20/10 $\mathrm{mmHg}$ within 10 minutes of assuming a standing position or during a head-up tilt on at least three separate occasions; and the absence of any disease that could account for symptoms of orthostatic intolerance. Patients with orthostatic intolerance were excluded from the investigation if they were smokers; were pregnant; had an uncontrolled thyroid or adrenal disorder; had a history of a systemic illness that could influence autonomic function, such as diabetes mellitus or systemic lupus erythematosus, cardiovascular disease, or drug or alcohol abuse; or had taken any drug that was metabolized by cytochrome $\mathrm{P} 450$ (CYP3A4) during the last 72 hours.

Study participants were requested to stop taking their medications and not drink caffeine-containing beverages, such as coffee, tea, or cola drinks, and caffeine-containing foods, such as chocolate, for at least 24 hours prior to testing. The participants were also asked to complete an autonomic nervous system questionnaire in order to obtain information on the type and extent of their POTS symptoms before and after testing. Anamnesis, a physical examination, and electrocardiography (ECG) were performed on all participants prior to testing.

\section{Experimental Protocol}

All measurements were made in each participant between 08.00 and 11.00 a.m. in a quiet, darkened, and air-conditioned room with an ambient temperature of $\sim 24^{\circ} \mathrm{C}$. The experimental protocol comprised six phases during which the BP and HR were continuously monitored. First, baseline measurements of the arterial BP using a finger plethysmograph (Nexfin, BMEYE, Amsterdam, Netherlands), HR using a three-lead ECG, and respiratory rate (RR) of each participant were made. Second, each participant rested in a supine position on a tilt table for 30 minutes. Third, the RR and BP were sampled for eight minutes by a $500 \mathrm{~Hz} \mathrm{~A} / \mathrm{D}$ converter whose output was transferred to a standard personal computer using a specialized software package. The data on HR and BP variability (HRV and BPV, respectively) from each participant were analyzed off-line using a locally developed software package. Baroreflex sensitivity of each participant was calculated from the time-domain of the beat-to-beat HR and BP. Fourth, standard autonomic testing of the parasympathetic and sympathetic nervous systems were performed in each participant. Specifically, testing of the parasympathetic nervous system comprised the Valsalva maneuver and deep breathing, and testing of the sympathetic nervous system comprised a static hand grip for three minutes, hyperventilation for one minute, and immersion of the hand in ice water for one minute. Fifth, the patient was then head-up tilted at $70^{\circ}$ for 20 minutes. Finally, the patient was instructed to rest in a supine position on a tilt table for 30 minutes. This protocol was repeated $60-80$ minutes after administering a single oral dose of $7.5 \mathrm{mg}$ ivabradine. 


\section{Pharmacology of Ivabradine}

Ivabradine was originally developed for the treatment of chronic stable angina pectoris. Ivabradine blocks the $\mathrm{I}_{f}$ channel (funny channel, $\mathrm{HCN} 2 / 4$ channel), which is a mixed $\mathrm{Na}^{+}-\mathrm{K}^{+}$inward current that causes hyperpolarization of the membrane and is highly expressed in the SA node and atrioventricular node. This channel is typically controlled by the sympathetic nervous system. The sympathetic and parasympathetic nervous systems cause an increase and a decrease, respectively, in the $\mathrm{Na}^{+}$inward current and results in either tachycardia or bradycardia. Currently, ivabradine is approved for use in Europe only for anginal syndromes and inappropriate sinus tachycardia syndrome.

The pharmacokinetics and pharmacodynamics of ivabradine have been extensively studied in animals and humans. Its bioavailability is $40 \%$, and its elimination half-time is about two hours. On first pass, approximately $50 \%$ of the drug is metabolized by CYP3A4. Its protein-binding capacity is approximately $70 \%$, and it is eliminated by the kidneys with preserved partial activity of its metabolite. Dose adjustment is not needed for patients whose glomerular filtration rate is less than $15 \mathrm{~mL} / \mathrm{min}$. Its $\mathrm{C}_{\max }$ is $8.8 \mathrm{ng} / \mathrm{mL}$, and its $\mathrm{t}_{\max }$ is 0.9 hours $\left(45^{-90}\right.$ minutes) for an oral dose of $5 \mathrm{mg}$. Maximal HR control is achieved by a $20 \mathrm{mg}$ oral dose. In this study, we used a single oral dose of $7.5 \mathrm{mg}$ for safety purposes. The main adverse effects of ivabradine are luminous phenomena (mainly a sensation of enhanced brightness with a fully maintained visual field), sinus bradycardia, first-degree atrioventricular blocks, ventricular extra systoles, dizziness, and/or blurred vision. Ivabradine is contraindicated in sick sinus syndrome and should not be taken concomitantly with $\mathrm{CYP}_{3} \mathrm{~A}_{4}$ inhibitors.

\section{Statistical Analysis}

All data were analyzed using Microsoft Office Excel and Prism version 5.5 (GraphPad Software Inc., La Jolla, CA, USA). Results are presented as mean \pm standard error of the mean, and statistical significance was set at $5 \%$. Parametric continuous data were analyzed using a paired $t$ test. Non-parametric continuous data were analyzed using the MannWhitney $U$ test. The HRV and BPV were extracted from the time and frequency domains of the beat-tobeat systolic BP and RR intervals using a locally developed software package.

\section{RESULTS}

In one year, we were able to recruit eight patients who met our inclusion criteria. Their general characteristics are depicted in Table 1. Three subjects were taking fludrocortisone, and six subjects were taking propranolol, the $\beta$-adrenoceptor antagonist (mean dose $17 \pm 2 \mathrm{mg} /$ day) at the time of recruitment. Propranolol administration was gradually discontinued before the day of testing.

Patients reported that a single oral dose of $7.5 \mathrm{mg}$ ivabradine attenuated the main orthostatic symptoms, such as dizziness, blurred vision, and palpitations. None of the participants reported adverse effects that were related to the test drug.

Ivabradine decreased the resting HR by $4 \pm 1$ bpm. After tilting for five minutes, the $\mathrm{HR}$ significantly decreased $(P<0.01)$ from $118 \pm 4 \mathrm{bpm}$ to $101 \pm 5 \mathrm{bpm}$. Interestingly, ivabradine did not change the BP when the subject was resting in a supine position and during the head-up tilt (Table 2).

Ivabradine did not affect the cardiovascular vagal tone indices: Valsalva's ratio (maximal increase (phase II)/maximal decrease (phase IV) in HR along the maneuver), time-domain analysis of the HR variability root mean square of the successive differences (RMSSD), and the high-frequency domain of RR intervals $\left(\mathrm{H} f_{\text {rri }}\right)$. The funny channel blocker did not influence cardiovascular sympathetic tone, as measured by the indices of the cardiovascular control of $\mathrm{HR}$ and systolic $\mathrm{BP}$, namely the low-frequency domain of RR interval and systolic BP changes ( $\mathrm{L} f_{\mathrm{rri}}$ and $\mathrm{L} f_{\mathrm{BP}}$, respectively) (Table 2). Ivabradine also did not change the $\mathrm{L} f_{\text {rri }} / \mathrm{H} f_{\text {rri }}$ ratio during rest and tilt, a measure of the sympathovagal balance of HR (Table 2).

Table 1. General Characteristics of the Eight Study Participants.

\begin{tabular}{l|l}
\hline \multicolumn{1}{|c|}{ Characteristic } & \multicolumn{1}{c}{ Detail } \\
\hline Age & $31 \pm 3$ years \\
Female/Male & $6 / 2$ \\
Body Mass Index & $27 \pm 1.5 \mathrm{~kg} / \mathrm{cm}^{2}$ \\
POTS Duration & $2.6 \pm 1$ years (median: 2 years) \\
\hline
\end{tabular}

POTS, postural tachycardia syndrome. 
Table 2. Hemodynamic and Indices of Autonomic Function Before and After Ivabradine.

\begin{tabular}{|l|c|c|c|c|}
\hline \multirow{2}{*}{ Parameter } & \multicolumn{2}{|c|}{ Baseline } & \multicolumn{2}{c|}{ Ivabradine } \\
\cline { 2 - 5 } & Rest & Tilt & Rest & Tilt \\
\hline Systolic BP $(\mathrm{bmH})$ & $70 \pm 3$ & $118 \pm 4$ & $66 \pm 2^{*}$ & $101 \pm 5^{*}$ \\
Diastolic BP $(\mathrm{mmHg})$ & $113 \pm 5$ & $114 \pm 4$ & $114 \pm 3$ & $115 \pm 4$ \\
Valsalva ratio & $68 \pm 3$ & $75 \pm 3$ & $67 \pm 3$ & $74 \pm 4$ \\
$\mathrm{RMSSD}$ & $1.43 \pm 0.1$ & - & $1.52 \pm 0.2$ & - \\
$\mathrm{L} f_{\text {rri }}$ & $35 \pm 4$ & $16 \pm 2$ & $28 \pm 6$ & $18 \pm 2$ \\
$\mathrm{H} f_{\text {rri }}$ & $715 \pm 160$ & $470 \pm 180$ & $790 \pm 150$ & $390 \pm 120$ \\
$\mathrm{~L} f_{\text {rri }} / \mathrm{H} f_{\text {rri }}$ ratio & $790 \pm 120$ & $180 \pm 80$ & $840 \pm 140$ & $210 \pm 90$ \\
$\mathrm{~L} f_{\mathrm{BP}}$ & $1.15 \pm 0.2$ & $3.9 \pm 1.0$ & $0.95 \pm 0.1$ & $4.1 \pm 1.2$ \\
\hline
\end{tabular}

* Significant differences between the baseline and the tilt.

$\mathrm{BP}$, blood pressure; bpm, beats per minute; $\mathrm{H} f_{\mathrm{rri}}$, RR intervals high-frequency domain; $\mathrm{HR}$, heart rate; $\mathrm{L} f_{\mathrm{BP}}$, BP lowfrequency domain; $L f_{\text {rri }}$, RR intervals low-frequency domain; RMSSD, root mean square of the successive differences.

\section{DISCUSSION}

The results of this investigation showed that a single oral dose of $7.5 \mathrm{mg}$ ivabradine significantly slowed the HR of POTS patients at rest and during tilting without any relevant adverse effects. Moreover, this dose of ivabradine had no effect on the indices of cardiovascular autonomic nervous system activity, especially the HRV.

Ivabradine is a pure HR-slowing agent, whose pharmacological target is the SA node. Hence, it is not altogether surprising that ivabradine slows the HR in POTS patients. This slowing of the HR can also be achieved following administration of $\beta$ adrenoceptor antagonists. 9 Since this slowing of the HR is associated with adverse effects such as fatigue, sleep disorders, and impotence, many POTS patients frequently discontinue treatment with $\beta$ adrenoceptor antagonists.

There are anecdotal reports that describe a therapeutically beneficial effect of ivabradine on POTS symptoms and HR reduction in POTS patients. Sutton et al. recently reported that ivabradine prevented syncope in patients with vasovagal syncope that is preceded by tachycardia.33,34 McDonald et al. reported their clinical observations on the efficacy, drug tolerance, and adverse effects of ivabradine in 22 POTS patients. 35 They found that ivabradine was well tolerated in most patients, and only a few adverse effects were reported. Six of these
22 patients discontinued the medication after 15 weeks due to the lack of a therapeutic benefit.

Our knowledge of the $\mathrm{I}_{f}$ channel, located in the SA node, is primarily based on animal studies. The activity of this channel is regulated by the autonomic nervous system (ANS). ${ }^{17}$ The sympathetic arm of the ANS activates the $\beta_{1}$-adrenoreceptor; its second messenger, cyclic AMP, increases $\mathrm{I}_{f}$ channel conductance. This increased conductance permits more $\mathrm{Na}^{+}$ions to move into the cells of the SA node. The HR increases due to the reduced polarity (phase IV of the action potential) of these cells. Parasympathetic or vagal tone of the heart is facilitated by M2 muscarinic receptors in the SA node. Stimulation of these receptors results in bradycardia by increasing the polarity of these cells in the SA node. Therefore, the sympathovagal balance of the SA node should affect $\mathrm{I}_{f}$ channel activity. We found that ivabradine did not change the sympathovagal balance, despite significant slowing of the HR. We also expected the indices of the vagal tone, namely the RMSSD and the $\mathrm{H} f_{\text {rri }}$, to increase with the simultaneous decrease in sympathetic tone. These results are therefore unexpected and we have no precise explanation. However, possible explanations include (1) the low dose of ivabradine that was given to our study patients, (2) that ivabradine was given as an acute single oral dose before testing, and testing was not done in POTS patients who had been using ivabradine for a prolonged period, (3) the early 
sampling of the ECG and BP (time-dependent effect), or (4) that the pharmacological action of ivabradine exerted on the funny channel may have spared the "outcome" of sympathovagal tone.

Joannides et al. recently reported that acute administration of ivabradine has no effect on ANS activity and function in healthy subjects, as measured by HRV analysis, despite slowing of the HR during rest and exercise. ${ }^{6}$ They also reported no evidence of a depressant effect on cardiac function. Nerla et al. also reported that the ivabradineinduced increase in vagal cardiovascular tone, in syncope patients, was very modest and less than that observed in patients who were given a $\beta$ adrenoceptor antagonist. 37

\section{CONCLUSIONS}

Ivabradine is a selective blocker of the $\mathrm{I}_{f}$ channel in cells of the SA node and is effective in slowing the HR of POTS patients at rest and during tilting, without producing significant adverse effects. Moreover, ivabradine exerts its effects without influencing the sympathovagal balance. In view of the limited information on the therapeutic benefits of ivabradine in POTS patients, there is a need to carry out additional investigations on its clinical effectiveness in a large group of POTS patients in a randomized double-blind placebo-controlled crossover trial.

\section{REFERENCES}

1. Furlan R, Jacob G, Snell M, et al. Chronic orthostatic intolerance: a disorder with discordant cardiac and vascular sympathetic control. Circulation 1998;98: 2154-9. Full Text

2. Grubb BP. Postural tachycardia syndrome. Circulation 2008;117:2814-17. Full Text

3. Low PA, Sandroni P, Joyner M, Shen WK. Postural tachycardia syndrome (POTS). J Cardiovasc Electrophysiol 2009;20:352-8. Full Text

4. Streeten DH. Orthostatic intolerance. A historical introduction to the pathophysiological mechanisms. Am J Med Sci 1999;317:78-87. Full Text

5. Jacob G, Shannon JR, Costa F, et al. Abnormal norepinephrine clearance and adrenergic receptor sensitivity in idiopathic orthostatic intolerance. Circulation 1999;99:1706-12. Full Text

6. Streeten DH, Scullard TF. Excessive gravitational blood pooling caused by impaired venous tone is the predominant non-cardiac mechanism of orthostatic intolerance. Clin Sci (Lond) 1996;90:277-85.
7. Bohora S. Joint hypermobility syndrome and dysautonomia: expanding spectrum of disease presentation and manifestation. Indian Pacing Electrophysiol J 2010;10:158-61.

8. Gazit Y, Nahir AM, Grahame R, Jacob G. Dysautonomia in the joint hypermobility syndrome. Am $\mathrm{J}$ Med 2003;115:33-40. Full Text

9. Jacob G, Costa F, Shannon JR, et al. The neuropathic postural tachycardia syndrome. N Engl J Med 2000; 343:1008-14. Full Text

10. Kanjwal K, Karabin B, Kanjwal Y, Grubb BP. Postpartum postural orthostatic tachycardia syndrome in a patient with the joint hypermobility syndrome. Cardiol Res Pract 2009;2009:187543. Full Text

11. Kanjwal K, Saeed B, Karabin B, Kanjwal Y, Grubb BP. Comparative clinical profile of postural orthostatic tachycardia patients with and without joint hypermobility syndrome. Indian Pacing Electrophysiol J 2010;10:173-8.

12. Keller NR, Robertson D. Familial orthostatic tachycardia. Curr Opin Cardiol 2006;21:173-9. Full Text

13. Rowe PC, Barron DF, Calkins H, Maumenee IH, Tong PY, Geraghty MT. Orthostatic intolerance and chronic fatigue syndrome associated with Ehlers-Danlos syndrome. J Pediatr 1999;135:494-9. Full Text

14. Shannon JR, Flattem NL, Jordan J, et al. Orthostatic intolerance and tachycardia associated with norepinephrine-transporter deficiency. N Engl J Med 2000;342:541-9. Full Text

15. Bohm M, Swedberg K, Komajda M, et al. Heart rate as a risk factor in chronic heart failure (SHIFT): the association between heart rate and outcomes in a randomised placebo-controlled trial. Lancet 2010; 376:886-94. Full Text

16. Colin $\mathrm{P}$, Ghaleh B, Monnet X, Hittinger L, Berdeaux A. Effect of graded heart rate reduction with ivabradine on myocardial oxygen consumption and diastolic time in exercising dogs. J Pharmacol Exp Ther 2004;308:236-40. Full Text

17. DiFrancesco D, Camm JA. Heart rate lowering by specific and selective I(f) current inhibition with ivabradine: a new therapeutic perspective in cardiovascular disease. Drugs 2004;64:1757-65. Full Text

18. Fox K, Ford I, Steg PG, Tendera M, Ferrari R. Ivabradine for patients with stable coronary artery disease and left-ventricular systolic dysfunction (BEAUTIFUL): a randomised, double-blind, placebocontrolled trial. Lancet 2008;372:807-16. Full Text

19. Gupta A, Sharma YP. Ivabradine and outcomes in chronic heart failure. Lancet 2010;376:2069-70. Full Text 
20. Kang SM, Won H, Hong N, Oh J. Ivabradine and outcomes in chronic heart failure. Lancet 2010;376:2069-70. Full Text

21. Monnet X, Colin P, Ghaleh B, Hittinger L, Giudicelli $\mathrm{JF}$, Berdeaux A. Heart rate reduction during exerciseinduced myocardial ischaemia and stunning. Eur Heart J 2004;25:579-86. Full Text

22. Reil JC, Bohm M. BEAUTIFUL results--the slower, the better? Lancet 2008;372:779-80. Full Text

23. Sulfi $\mathrm{S}$, Timmis AD. Ivabradine -- the first selective sinus node I(f) channel inhibitor in the treatment of stable angina. Int J Clin Pract 2006;60:222-8. Full Text

24. Swedberg K, Komajda M, Bohm M, et al. Ivabradine and outcomes in chronic heart failure (SHIFT): a randomised placebo-controlled study. Lancet 2010;376:875-85. Full Text

25. Fox K, Ford I, Steg PG, et al. Ivabradine in stable coronary artery disease without clinical heart failure. N Engl J Med 2014;371:1091-9. Full Text

26. Tardif JC, Ford I, Tendera M, Bourassa MG, Fox K. Efficacy of ivabradine, a new selective I(f) inhibitor, compared with atenolol in patients with chronic stable angina. Eur Heart J 2005;26:2529-36. Full Text

27. Bois P, Bescond J, Renaudon B, Lenfant J. Mode of action of bradycardic agent, $S$ 16257, on ionic currents of rabbit sinoatrial node cells. $\mathrm{Br} \mathrm{J}$ Pharmacol 1996;118:1051-7. Full Text

28. DiFrancesco D. Funny channels in the control of cardiac rhythm and mode of action of selective blockers. Pharmacol Res 2006;53:399-406. Full Text

29. DiFrancesco D, Borer JS. The funny current: cellular basis for the control of heart rate. Drugs 2007;67(Suppl 2):15-24. Full Text
30. DiFrancesco D. The role of the funny current in pacemaker activity. Circ Res 2010;106:434-46. Full $\underline{\text { Text }}$

31. Thollon C, Cambarrat C, Vian J, Prost JF, Peglion JL, Vilaine JP. Electrophysiological effects of S 16257, a novel sino-atrial node modulator, on rabbit and guinea-pig cardiac preparations: comparison with UL-FS 49. Br J Pharmacol 1994;112:37-42. Full Text

32. Thollon C, Bedut S, Villeneuve $\mathrm{N}$, et al. Usedependent inhibition of hHCN4 by ivabradine and relationship with reduction in pacemaker activity. $\mathrm{Br}$ J Pharmacol 2007;150:37-46. Full Text

33. Sutton R, Salukhe T. Ivabradine in the treatment of orthostatic intolerance. Europace 2011;13:306-7. Full Text

34. Sutton R, Salukhe TV, Franzen-McManus AC, Collins A, Lim PB, Francis DP. Ivabradine in treatment of sinus tachycardia mediated vasovagal syncope. Europace 2014;16:284-8. Full Text

35. McDonald C, Frith J, Newton JL. Single centre experience of ivabradine in postural orthostatic tachycardia syndrome. Europace 2011;13:427-30. Full Text

36. Joannides R, Moore N, Iacob M, et al. Comparative effects of ivabradine, a selective heart rate-lowering agent, and propranolol on systemic and cardiac haemodynamics at rest and during exercise. Br J Clin Pharmacol 2006;61:127-37. Full Text

37. Nerla R, Di FA, Milo M, et al. Differential effects of heart rate reduction by atenolol or ivabradine on peripheral endothelial function in type 2 diabetic patients. Heart 2012;98:1812-16. Full Text 